\title{
FINITE NEAR-RINGS WITH TRIVIAL ANNIHILATORS
}

\section{GEORGE SZETO}

(Received 18 April 1972)

Communicated by G. E. Wall

\section{Introduction}

In [3] and [4], the near-rings $R$ with no zero divisors are studied. In particular, a near-ring $R$ is a near-field if it has a non-zero right distributive element ([4], Theorem 1.2.). Also, $(R,+)$ is a nilpotent group if not all non-zero elements of $R$ are left identities of $R$ ([3], Theorem 2). The purpose of the present paper is to extend the above results to a class of near-rings with zero divisors; that is, the set of annihilators of an element $x$ in $R, T(x)=\{g / x g=0\}$ is either $\{0\}$ or $R$. The examples of such near-rings are those $R$ with $(R,+)$ simple groups and those $R$ with no zero divisors as given in [1], [2], [3] and [4]. For this $R$, we can easily see that $R=A \cup S$ where $A=\{x / T(x)=R\}$ and $S=\{x / T(x)$ $=\{0\}\}$. Then the second part of this paper will give a structural theorem on the semi-group $(S, \cdot)$, and more properties on $R$ can be derived.

Throughout the present paper $(R,+, \cdot)$ is assumed a finite near-ring such that for each $x$ in $R, T(x)=\{y / x y=0\}$ is either $R$ or $\{0\}$. If $T(0)=\{0\}$ then each $0 a \neq 0$ for each $a \neq 0$ in $R$. But then $0 R=R$; and so $0 a$ is a left identity of $R$ for each $a \neq 0$. From now on $R$ is assumed not this kind just mentioned. So, $R$ has the property that $T(0)=R$.

\section{2}

Section 1. Assume $T(x)$ is either $R$ or $\{0\}$ for each $x$ in $R$, we shall show that either the multiplication operation on $R$ is trivial (that is, for $r \neq 0$ in $R$, $r g=0$ for all $g$ in $R$ or $r g=g$ for all $g$ in $R$ ), or the additive group $(R,+)$ is nilpotent. This extends Theorem 2 in [3].

LeMma 1.1. Let $S=\{x / T(x)=\{0\}\}$ and let $A=\{x / T(x)=R\}$; then (1) $R=A \cup S$ such that $A \cap S$ is a void set and (2) $s S=S$ and $s A=A$ for each $s$ in $S$, where $s A=\{$ sa for $a$ in $A\}$ and $s S=\{$ st for $t$ in $S\}$. 
PrCof. For each $x$ in $R T(x)$ is either $R$ or $\{0\}$, so part (1) is trivial. Next for each $a$ in $A s a R=s(a R)=s 0=0$, so $s A \subset A$. Let $x$ be an element in $S$ and $r$ in $R$ such that $s x r=0$. Then $s(x r)=0, x r=0$; and so $r=0$. Thus $s S \subset S$. On the other hand, for any $r^{\prime}$ and $r^{\prime \prime}$ in $R$ such that $s r^{\prime}=s r^{\prime \prime}$, we have $s\left(r^{\prime}-r^{\prime \prime}\right)=0$. Hence $r^{\prime}=r^{\prime \prime}$ because $T(s)=\{0\}$. Therefore $s A=A$ and $s S=S$.

LemMA 1.2. By keeping the notations of lemma 1.1, if $s t=t$ for some elements $s$ and $t$ in $S$, then $s r=r$ for each $r$ in $R$.

Proof. Since $t S=S$ and $t A=A$ by lemma 1.1, $t R=R$; and so for each $r$ in $R, r=t r^{\prime}$ for some $r^{\prime}$ in $R$. Hence $s r=s\left(t r^{\prime}\right)=(s t) r^{\prime}=t r^{\prime}=r$.

Using similar idea to [3] we can show our main theorem in this section.

THEOREM 1.3. By keeping the notations of Lemma 1.1, we have

(1) if $T(x)=R$ for each $x$ in $R$ then $R^{2}=\{0\}$;

(2) if $T(x)=\{0\}$ with some $x \neq 0$ and if $A$ contains no non-zero subgroups of $(R,+)$; then either $x$ is a left identity of $R$ or $(R,+)$ is a nilpotent group.

Proof. Part (1) is trivial by the definition of $T(x)$. Next, since $T(x)=\{0\}$ with some $x \neq 0$ in $R, x$ is in $S$; and so $x R=R$ by lemma 1.1. Suppose $x$ is not a left identity of $R$. Then $x^{2} \neq x$. For otherwise $x r=r$ for each $r$ in $R$ by lemma 1.2. This contradicts that $x$ is not a left identity. Hence we can have the identity $x^{n}$ of the cyclic group generated by $x$ under multiplication with $n>1$; that is $x^{n} x=x x^{n}$ with a minimal integer $n$. Again since $x R=R, x^{n}(x r)$ is equal to $x r$ for each $x r$ in $x R$; so $x^{n}$ is a left identity of $R$ with $n>1$. Futhermore, it is not hard to show that $\alpha_{y}$ defined by $\alpha_{y}(r)=y r$ for each $r$ in $R$ is a group automorphism of $(R,+)$ if $y$ is in $S$. Since $n>1, n=p m$ for some prime integer $p$ and an integer $m$. Noting that the element $x^{m}$ has order $p$, and that $x^{m}$ is in $S$, we have that $\alpha_{\left(x^{m}\right)}$ is an automorphism of $(R,+)$ of order $p$. Also, $\alpha_{\left(x^{m}\right)}$ is a fixed point free automorphism. In fact, let $\alpha_{\left(x^{m}\right)}(r)=r$, that is, $x^{m} r=r$. Then there are two cases.

Case 1. $r$ is in $S$. Then $r R=R$ by Lemma 1.2; and so $x^{m}$ is a left identity of $R$. Thus $x^{m} x=x$, a contradiction to the minimal property of $n$ such that $x^{n} x=x$. This implies that $\alpha_{\left(x^{m}\right)}$ is a fixed point free automorphism of order $p$. Therefore $(R,+)$ is a nilpotent group by [6].

Case 2. $r$ is in $A$. Let $C=\left\{h / h\right.$ in $R$ and $\left.x^{m} h=h\right\}$. Then 0 and $r$ are in $C$. For each $h^{\prime}$ and $h^{\prime \prime}$ is $C, x^{m}\left(h^{\prime}-h^{\prime \prime}\right)=x^{m} h^{\prime}-x^{m} h^{\prime \prime}=h^{\prime}-h^{\prime \prime}$. Hence $(C,+)$ is a subgroup of $(R,+)$. Noting that $C$ can be assumed a subset of $A$. For otherwise there exists $h$ in $S$ such that $x^{m} h=h$; and so this leads to case 1 . But by hypothesis the set $A$ has no non-zero subgroup of $(R,+)$, so $C=\{0\}$. Hence $r=0$. Thus $\alpha_{\left(x^{m}\right)}$ is a fixed point free automorphism of order $p$; and so $(R,+)$ is nilpotent. 
A near-ring $R$ is a near-integral domain if and only if $A=\{0\}$ and not all elements of $R$ are left identities ([3], Def. Section 2). Then our theorem extends the Theorem 2 in [3].

COROLlary 1.4. (Ligh) Let $R$ be a finite near-integral domain. Then $(R,+)$ is nilpotent.

From $R=A \cup S$ with $A \cap S$ a void set, it can be shown that a trivial multiplication on $A$ implies a trivial multiplication on $R$.

PROPOSITION 1.5. If $(S \cup\{0\},+)$ is a proper subgroup of $(R,+)$ and if $s a=a$ for each $a$ in $A$ and some $s$ in $R$ (and so in $S$ ), then $s r=r$ for each $r$ in $R$.

Proof. For any element $t$ in $S, a+t$ is not in $S$ because $(S \cup\{0\},+)$ is a subgroup of $(R,+)$ and because $a$ is not in $S \cup\{0\}$. Hence $s(a+t)=a+t$. But then $s a+s t=a+s t=a+t, s t=t$ for each $t$ in $S$. Thus $s r=r$ for each $r$ in $R$.

Since for each $s$ in $S, \alpha_{s}$ defined by $\alpha_{s}(r)=s r$ is a group automorphism of $(R,+)$. Hence from Proposition 1.5 we have:

COROLLARY 1.6. If $(S \cup\{0\},+)$ is a subgroup of $(R,+)$ and if the automorphism $\alpha_{s}$ has at least two non-zero fixed points in a same coset of $(S \cup\{0\},+)$; then $\alpha_{s}$ is an identity automorphism.

Proof. Let $a^{\prime}$ and $a^{\prime \prime}$ be two non-zero fixed points of $\alpha_{s}$ in a same coset of $(S \cup\{0\},+)$ in $R$. Then $a^{\prime}=a^{\prime \prime}+r$ for some $r$ in $S$ such that $\alpha_{s}\left(a^{\prime}\right)=a^{\prime}$ and $\alpha_{s}\left(a^{\prime \prime}\right)=a^{\prime \prime}$; and so

$$
\alpha_{s}\left(a^{\prime}\right)=\alpha_{s}\left(a^{\prime \prime}+r\right)=a^{\prime \prime}+r .
$$

But $\alpha_{s}\left(a^{\prime \prime}+r\right)=\alpha_{s}\left(a^{\prime \prime}\right)+\alpha_{s}(r)$ then $\alpha_{s}\left(a^{\prime \prime}+r\right)=a^{\prime \prime}+\alpha_{s}(r)=a^{\prime \prime}+r$. Hence $\alpha_{s}(r)=r$; that is, $s r=r$. This implies that $s t=t$ for all $t$ in $R$ by Lemma 1.2. Thus $\alpha_{s}$ is the identity automorphism of $(R,+)$.

(3) Section 2. By Lemma 1.1, $R=A \cup S$, so, in case $S$ is a void set, we have $R^{2}=\{0\}$, and in case $A=\{0\}$, we have a near-integral domain. In this section, $S$ is always assumed non-void. We shall give the following structural theorem on the semi-group $(S, \cdot): S$ is partitioned as isomorphism multiplicative groups. Consequently, some of the results of [4] can be extended.

LEMMA 2.1. For each element $s$ in $S$, it has a unique right identity $s$ ' which is also a left identity of $R$.

Proof. Since $T(s)=\{0\}$ and since $R$ is finite, there is a multiplicative group generated by $s$ of order $n$,

$$
\left\{s=s^{n+1}, s^{2}, \cdots, s^{n}\right\} .
$$


Hence $s^{n}$ is a right identity of $s$. Suppose $t$ is also a right identity of $s$. Then $s t=s s^{n}$; and so $s\left(t-s^{n}\right)=0$. Thus $t=s^{n}=s^{\prime}$ because $T(s)=\{0\}$ again. This implies that $s^{\prime}$ is unique. Moreover, noting that $s^{\prime} s=s^{n} s=s$ and that $s R=R$ we conclude that $s^{\prime}=s^{n}$ is a left identity of $R$ by Lemma 1.2 .

Definition. For any $x$ and $y$ in $S$, we call $x$ equivalent to $y$ if and only if the identity of $x=$ the identity of $y$.

THEOREM 2.2. (a) The relation " $\sim$ " defined above on $S$ is an equivalence relation;

(b) Each equivalence class of " $\sim ", R_{x}=\{y /$ the identity of $y=$ the identity of $x\}$, is a multiplicative group;

are isomorphic.

(c) Any two equivalence classes, $R_{x}$ and $R_{y}$, for $x$ and $y$ in $S$,

Proof. Part (a) is obvious. For part (b), let $a$ and $b$ be in $R_{x}$ with the right identity $x^{n}$. Then $(a b) x^{n}=a\left(b x^{n}\right)=a b$. Also, $a a^{k-1}=a^{k}=x^{n}$, where $k$ is the order of $a$, so $a^{k-1}=a^{-1}$. Hence $R_{x}$ is a multiplicative group with the identity $x^{n}$. Finally for part (c), let $R_{x}$ and $R_{y}$ be any two equivalence classes with the right identities $x^{\prime}$ and $y^{\prime}$ respectively. Define a map $\beta$ from $R_{x}$ to $R_{y}$ by $\beta\left(r x^{\prime}\right)$ $=\left(r x^{\prime}\right) y^{\prime}$ for each $r x^{\prime}$ in $R_{x}$ (for $R_{x}=S_{x}{ }^{\prime}$ ). Since $x^{\prime}$ is also a left identity of $R$ by Lemma 2.1 ,

$$
\beta\left(r x^{\prime}\right)=\left(r x^{\prime}\right) y^{\prime}=r\left(x^{\prime} y^{\prime}\right)=r y^{\prime} .
$$

We claim that $\beta$ is a group isomorphism from $R_{x}$ onto $R_{y}$. In fact, for any $a x^{\prime}$ and $b x^{\prime}$ in $R_{x}$,

$$
\begin{aligned}
\beta\left(a x^{\prime} b x^{\prime}\right)= & \left.\beta\left(a b x^{\prime}\right)=\left(a b y^{\prime}\right)=(a b) y^{\prime}=\left(a y^{\prime}\right)\left(b y^{\prime}\right)\right)= \\
& \left(a x^{\prime} y^{\prime}\right)\left(b x^{\prime} y^{\prime}\right)=\beta\left(a x^{\prime}\right) \beta\left(b x^{\prime}\right)
\end{aligned}
$$

by Lemma 2.1. again. Next let $\beta\left(a x^{\prime}\right)=y^{\prime}$ for an element $a x^{\prime}$ in $R_{x}$, then $\left(a x^{\prime}\right) y^{\prime}$ $=y^{\prime}$. Since $y^{\prime} R=R, a x^{\prime}$ is a left identity of $R$; and so $a x^{\prime}$ is the identity of the multiplicative group $R_{x}$. Hence $a x^{\prime}=x^{\prime}$. Thus $\beta$ is one to one. Furthermore, let $a y^{\prime}$ be an element in $R_{y}$, then $a x^{\prime}$ is in $R_{x}$ such that

$$
\beta\left(a x^{\prime}\right)=\left(a x^{\prime}\right) y^{\prime}=a y^{\prime} .
$$

This implies that $\beta$ is onto and therefore the theorem is proved.

The following consequences are immediate.

COROLlaRY 2.3. The number of elements in $S=\left(\right.$ order of $R_{x}$ ) times (the number of equivalence classes of " "). 
COROLLARY 2.4. The following statements are equivalent:

(a) $R_{x} \cup\{0\}$ is a subnear-ring of $(R,+, \cdot)$;

(b) $R_{x} \cup\{0\}$ is a near-field;

(c) $\left(R_{x} \cup\{0\},+\right)$ is a subgroup of $(R,+)$.

Proof. Since $R_{x}$ is a multiplicative group, the proof is trivial.

In theorem 1.3, we assumed that the set $A$ contains no non-zero subgroups of $R$ under addition ([1], 2-3, example 6). But if $R$ has a non-zero right distributive element, then this assumption does not hold.

THEOREM 2.5. If $R$ has a non-zero right distributive element, then $(A,+)$ is a normal subgroup of $(R,+)$.

Proof. Let $x$ be a non-zero right distributive element in $R$. Then for any elements $a$ and $b$ in $A(a+b) x=a x+b x=0$; and so $a+b$ is in $A$. Hence $(A,+)$ is a subgroup of $(R,+)$. Moreover, for each $c$ in $R,(-c+a+c) x$ $=(-c) x+a x+c x=-(c x)+0+c x=0$ because $x$ in $a$ right distributive element. Thus $(A,+)$ is normal in $(R,+)$.

REMARK 1. The near-ring $R$ under consideration is $S \cup A$ by Lemma 1.1. From the definitions of $A$ and $S$, we know that $A-\{0\}$ is the set of left zero divisors of $R$ and that $S$ is the set of elements without right zero divisors. Hence if $R$ has a right distributive non-zero elements, then the number of elements of $R$ is less than $n^{2}$ where $n+1$ is the order of the normal subgroup $(A,+)$ in Theorem 2.5 ([4], Th. 2.3).

REMARK 2. If $R$ has a right distributive element in $S$ with $S \cup 0$ a group under + then $S$ has only one equivalence class in the sense of Theorem 2.2 . $S$ is equal to the class, so it is a multiplicative group. $S \cup\{0\}$ is a near-ring, for $(S \cup\{0\},+)$ is a subgroup of $(R,+)$. This implies that $R=S \cup\{0\}$ because. the complement of $S,(A,+)$, is also a subgroup of $(R,+)$ by Theorem 2.5 . Thus this leads to Theorem 1.2. of [4]; and so $R$ is a near-field. After this paper had been submitted, the author learned that Theorem 2.2 for planar integral domains had been proved by J. Clay.

\section{References}

[1] J. Clay, 'The near-rings on groups of low order', Math. Z. 104 (1968), 364-371.

[2] A. Fröhlich, The near-ring generated by the inner automorphisms of a finite simple group', J. London Math. Soc. 33 (1958), 95-107.

[3] S. Ligh, 'On the additive groups of finite near-integral-domains and simple D. G. nearrings', Monatsh. Math. 16 (1972), 317-322. 
[4] S. Ligh and J. Malone, 'Zero divisors and finite near-rings', J. Austral. Math. Soc., Vol. 11 (1970), 374-378.

[5] J. Malone and C. Lyons, 'Endomorphism near-rings', Proc. Edinburgh Math. Soc. 17 (1970), 71-78.

[6] J. Thompson, 'Finite groups with fixed-point-free automorphisms of prime order', Proc. Natl. Acad. Sci., U. S. A. 45 (1959), 578-581.

Mathematics Department

Bradley University

Peoria, Illinois 61606

U. S. A. 